ISBN 978-981-14-1684-2

Proceedings of 2019 the 9th International Workshop on Computer Science and Engineering

(WCSE 2019 SUMMER)

Hong Kong, 15-17 June, 2019, pp. 207-212

doi: $10.18178 /$ wcse.2019.06.031

\title{
Research on Visual Effect of Enhancing Image
}

\author{
Chao Huang $^{1+}$ \\ ${ }^{1}$ School of Information Science and Engineering, Zaozhuang University, Zaozhuang, 277160, China
}

\begin{abstract}
The visual effect of an image is affected by many factors. In order to get a better visual effect, it is necessary to pre-process the collected image to improve the number of image feature points matched. In this paper, histogram equalization, homomorphic filtering based on illumination-reflection model and enhancement method based on Retinex theory are utilized to pre-process the collected images, so as to enhance the contrast of the images. And Scale Invariant Feature Transform (SIFT), Speeded Up Robust Features (SURF) and Affine Scale Invariant Feature Transform (ASIF) are applied to extract and match the image features of the pre-processed images. The results show that the number of feature points of the image processed by enhancement methods is much higher than that of the image without enhancement.
\end{abstract}

Keywords: histogram equalization, homomorphic filter, Retinex, enhancement

\section{Introduction}

Image matching is to transform several images acquired by the same sensor under different conditions, or under different shooting conditions, different sensors and different perspectives, so that the same scene of the image can complete the process of pixel level position matching. Image features include point features, line features, surface features, edge features, and moment features. Corner points and high curvature points are classified as point features, while edge lines, wide baselines, line endpoints, line intersections and curvature discontinuities are classified as line features. Image feature matching is a basic method in computer vision research. In photogrammetry, image processing [1-2], remote sensing image, 3D reconstruction [3], computer vision [4], and other related research fields, image feature matching is a hot research topic. The purpose of feature matching is to search the corresponding features in the same scene, such as point features or line features. However, the changes of illumination and perspective make feature matching a challenging task. In recent years, significant progress has been made in the field of feature matching, and a number of algorithms have been proposed [5-6].

Due to the influence of imaging conditions, such as camera position, focal length, illumination and attitude changes, and sensor performance, the same area of the collected images is not only different in appearance on different images, but also has projective distortion among the corresponding areas of the images [7-8]. And the extraction and matching of feature points from collected images will seriously affect the accuracy of extraction and matching. To solve this problem, it is necessary to pre-process the image in order to enhance the quality of the image. The purpose of image preprocessing is to remove the irrelevant information and restore the real information in the image, so that the information of the image can be easily detected and the quality of the image can be enhanced. This preprocessing process simplifies the data and improves the reliability of feature extraction, matching and recognition. In this paper, histogram equalization, homomorphic filtering based on illumination-reflection model and enhancement method based on Retinex theory are used to pre-process the collected images, so as to enhance the contrast of the images. Then, Scale Invariant Feature Transform (SIFT) [8], Speeded Up Robust Features (SURF) [9] and Affine Scale Invariant Feature Transform (ASIF) [10] algorithms are applied to extract and match image features.

\footnotetext{
+ Corresponding author. Tel.: + 13963256729.

E-mail address: 305331562@qq.com.
} 


\section{Methods of Image Enhancement}

Image feature points are the significant points in the image, and could be utilized to identify objects accurately in the image. Before extracting image feature points, the image is preprocessed in order to eliminate image blurring, reduce the interference of highlighted image noise, and help to extract stable feature points. Three methods can be utilized for image preprocessing.

\subsection{Enhancement method based on histogram equalization}

Histogram equalization is a non-linear process, and a contrast adjustment method of image histogram. Its purpose is to obtain a wider and more uniform distribution. This method is more suitable for human visual analysis. By using this method, the region with lower contrast can get higher contrast, which could enhance the brightness of the image. This method is especially suitable for the case that the image presents a very compact pixel contrast [12]. Through this adjustment, the gray level of the image pixels will have a ratio on the histogram without affecting the overall contrast. The realization of histogram equalization depends on the scheme that can effectively extend the most commonly used gray value distribution of pixels. Histogram equalization method can use multiple histograms to adjust the local contrast rather than the overall contrast. This method is called adaptive histogram equalization. The idea of adaptive histogram equalization is to perform a separate histogram equalization operation for different image regions in order to achieve the adjustment of local image contrast.

Histogram equalization is not to change the distribution of histogram, but to adjust the contrast of image, so that the distribution of histogram is more balanced. Histogram equalization is a special case of histogram mapping technology. For gray-scale images, histogram equalization method can be regarded as a transformation, which could create a new image, so that the cumulative histogram of the new image changes linearly in the range of values.

From the image processing effect, the contrast of the image is obviously enhanced, and the gray distribution of the processed image is more uniform. Histogram equalization can achieve a satisfactory image effect. It makes full use of the frequency information of the image, and separates the high frequency part from the low frequency part of the image, so that the image can achieve a more attractive balance and retain more original gray image distribution.

\subsection{Homomorphic filtering enhancement method based on illumination-reflection model}

The principle of homomorphic filtering based on illumination-reflection model is to estimate the lowfrequency or high-frequency components of the image by filtering function, enhance the local contrast of the image, and compress the dynamic range of the image.

Homomorphic filtering of image belongs to the range of image processing in frequency domain. The problem of uneven illumination of the image can be eliminated, and the gray scale of the image is adjusted. Then the image details of the dark area are enhanced. At the same time, the details of the bright area of the image are preserved. The image $I(x, y)$ can be represented by illumination component $L(x, y)$ and reflection component $R(x, y)$. Illumination component $L(x, y)$ describes the illumination of the scene, which is independent of the scene. Reflection component $R(x, y)$ includes the details of the target, which is independent of the illumination. Its expression is:

$$
I(x, y)=L(x, y) \times R(x, y)
$$

Where $0<L(x, y)<\infty, 0<R(x, y)<1$.

The flowchart of homomorphic filtering is as follows.

$$
I(x, y) \rightarrow \log (\mathrm{I}) \rightarrow D F T \rightarrow H(u, v) \rightarrow(D F T)^{-1} \rightarrow \exp \rightarrow O(x, y)
$$

Where image $I(x, y)$ is represented by the product of reflection component and illumination component, which is $I(x, y)=L(x, y) \times R(x, y) . \log (I)$ is the logarithm of $I$. DFT represents Fourier transform for data, $H(u, v)$ denotes the filtering for data, $(D F T)^{-1}$ is inverse Fourier transform, and $O(x, y)$ denotes the image data after processing. 


\subsection{Enhancement method based on Retinex theory}

The composition of the image is shown in Fig. 1. The image is composed of two main parts: the incident light and the reflected object.

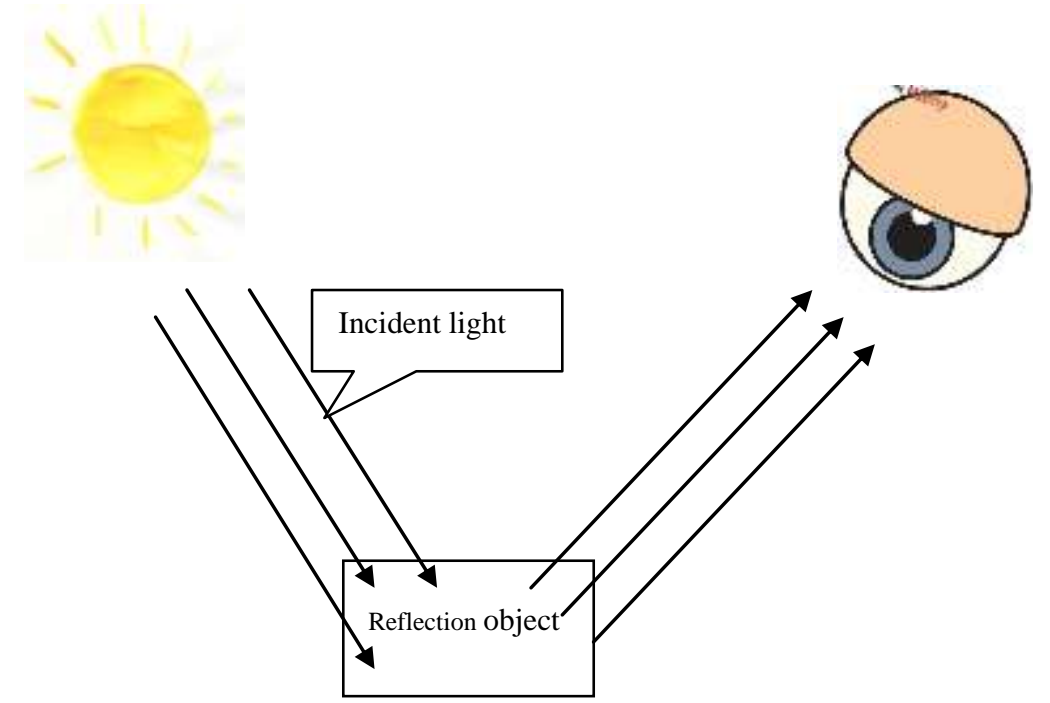

Fig. 1: Image formation process.

The image $I(x, y)$ is decomposed into the reflective image $R(x, y)$ and the brightness image $L(x, y)$ of the object, whose expression is $I(x, y)=R(x, y) \bullet L(x, y)$. The intrinsic nature of the image is determined by $R(x, y)$ and the dynamic range of the image pixels is determined by $L(x, y)$. An image is decomposed into two parts: brightness image and reflection image, which is the basic principle of Retinex algorithm. By reducing the influence of brightness image on reflected image, Retinex algorithm achieves the purpose of image enhancement. In order to facilitate the calculation, the logarithmic treatment is applied this algorithm. Logarithmic form is closer to the perception ability of human eye brightness, and makes complex product forms to transform into simple addition and subtraction forms.

$$
\log I(x, y)=\log (R(x, y) \bullet L(x, y))=\log R(x, y)+\log L(x, y)
$$

The enhanced modeling is described as follows.

$$
\log R(x, y)=\log \frac{I(x, y)}{L(x, y)}=\log I(x, y)-\log (I(x, y) \bullet G(x, y))
$$

Where $G(x, y)$ represents the low-pass convolution function, which is utilized to estimate the brightness $L(x, y)$ from the input image. At the same time, the local area of the input image is processed by Gaussian convolution function, which can better enhance the image. Gaussian convolution function is $G(x, y)=\lambda \bullet e^{-\frac{x^{2}+y^{2}}{c^{2}}}$, where $c$ is a scale constant. The smaller $c$ is, the more dynamic range of gray level will be compressed; on the contrary, the larger $c$ is, the sharper the image will be. $\lambda$ is a constant matrix, which makes $\iint G(x, y) d x d y=1$, in order to obtain the enhanced image.

Land's Retinex algorithm is essentially an image enhancement algorithm based on illumination compensation. The flow chart of the algorithm is shown in Fig. 2.

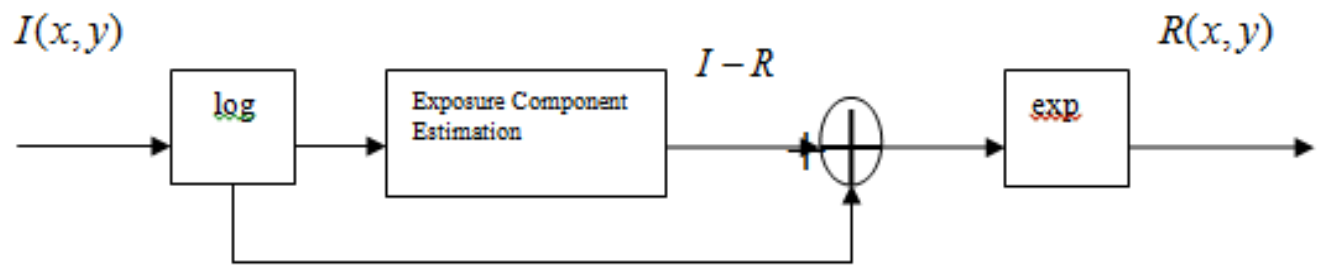

Fig. 2: The flowchart of image enhancement based on Retinex method. 
The core part of Retinex algorithm is the estimation of irradiation component. MSR algorithm is adopted as the estimation of irradiation component. Retinex algorithm has obvious advantages, which can restore most of the details of the original image and avoid the polarization trend caused by histogram equalization algorithm. The quality of the enhanced image is softer, and the visual effect is better. Retinex algorithm can not only compress the dynamic range, but also enhance the edge of the image, so as to achieve mutual coordination and maintain the color constancy of the original image. Retinex algorithm also overcomes the comprehensive application of various enhancement methods, which leads to the shortcomings of image enhancement effect.

The results of the enhancement method based on Retinex theory are shown in Fig. 3. As can be seen from Fig. 3, the illumination of the image is compensated, and the visual effect of the enhanced image is better. In Retinex algorithm, the illumination compensation method is utilized to approximate the reflected image of the original image, and the brightness image is estimated by using Gaussian smoothing function. When using illumination estimation, it is assumed that the illumination in the image is smooth.
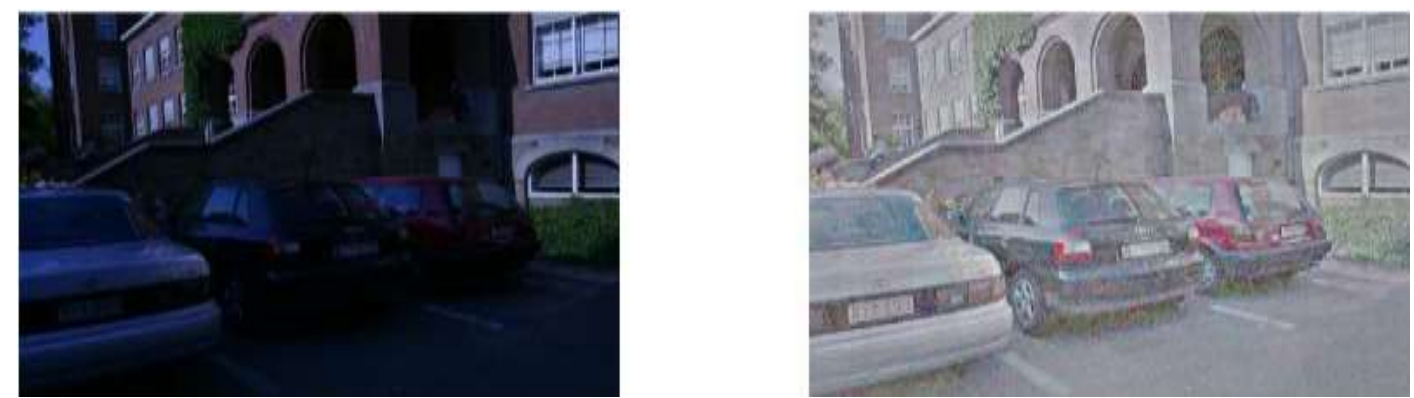

Fig. 3: Comparison of between original image and image processed by Retinex method.

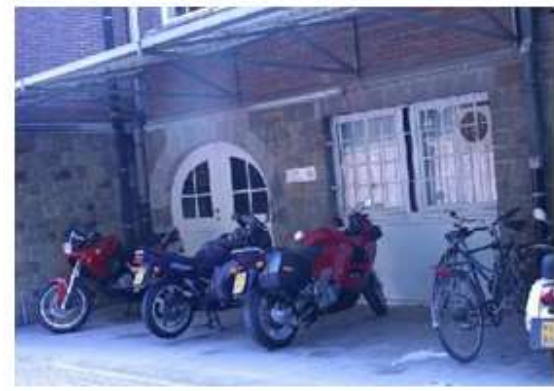

A

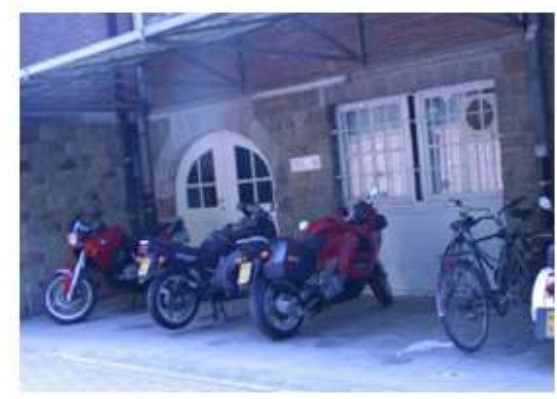

C

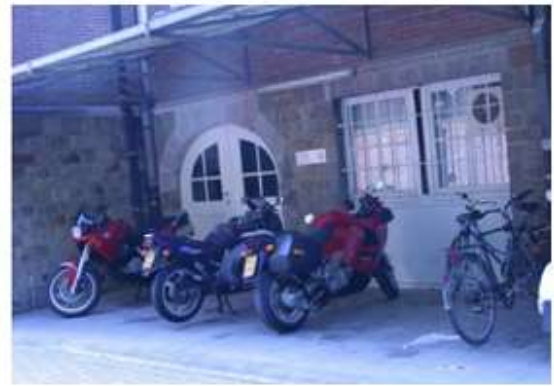

B

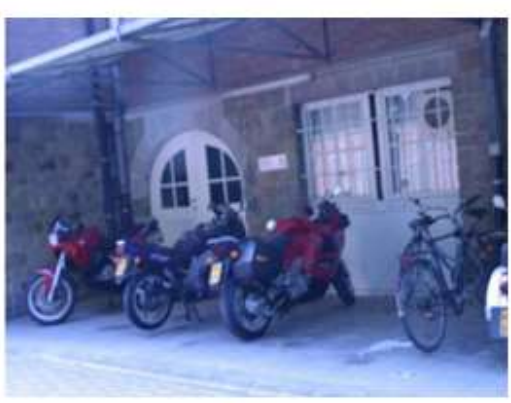

$\mathrm{D}$

Fig. 4: Blurred image.

\section{Experiments}

Aiming at the visual effect of poor image quality caused by image blurring, the three methods are utilized to process the blurred images, which are depicted in Fig. 4. Image A is taken as the reference image, and the other three images are taken as the image to be matched. From Fig. 4, it could be seen that the visual effect of the image gradually becomes blurred from A to D. In order to compare clearly, these images are divided into three pairs: A and B, A and C, A and D. The experimental results are listed in Tables 1, Table 2 and Table 3, respectively. 
Table 1: Matching table of A and B characteristic points in blurred images.

\begin{tabular}{|c|c|c|c|}
\hline & SIFT & SURF & ASIFT \\
\hline Unprocessed image & 148 & 210 & 5361 \\
\hline $\begin{array}{c}\text { Image processed by } \\
\text { histogram equalization }\end{array}$ & 176 & 356 & 7174 \\
\hline $\begin{array}{c}\text { Image processed by } \\
\text { homomorphic filtering }\end{array}$ & 110 & 201 & 5102 \\
\hline $\begin{array}{c}\text { Image processed by } \\
\text { Retinex method }\end{array}$ & 43 & 46 & 2488 \\
\hline
\end{tabular}

Table 2: Matching table of $\mathrm{A}$ and $\mathrm{C}$ characteristic points in blurred images.

\begin{tabular}{|c|c|c|c|}
\hline & SIFT & SURF & ASIFT \\
\hline Unprocessed image & 149 & 207 & 5444 \\
\hline $\begin{array}{c}\text { Image processed by } \\
\text { histogram equalization }\end{array}$ & 192 & 359 & 7025 \\
\hline $\begin{array}{c}\text { Image processed by } \\
\text { homomorphic filtering }\end{array}$ & 125 & 209 & 5075 \\
\hline $\begin{array}{c}\text { Image processed by Retinex } \\
\text { method }\end{array}$ & 50 & 45 & 2409 \\
\hline
\end{tabular}

Table 3: Matching table of A and D characteristic points in blurred images.

\begin{tabular}{|c|c|c|c|}
\hline & SIFT & SURF & ASIFT \\
\hline Unprocessed image & 142 & 194 & 20316 \\
\hline $\begin{array}{c}\text { Image processed by } \\
\text { histogram equalization }\end{array}$ & 180 & 335 & 31785 \\
\hline $\begin{array}{c}\text { Image processed by } \\
\text { homomorphic filtering }\end{array}$ & 122 & 184 & 19222 \\
\hline $\begin{array}{c}\text { Image processed by Retinex } \\
\text { method }\end{array}$ & 57 & 48 & 19089 \\
\hline
\end{tabular}

From Table 1, Table 2 and Table 3, it could be seen that the correct matching points of the image which is processed by histogram equalization, extraction and matching of the feature points are obviously higher than those of the image without being processed. Especially, the change of the feature points matched by ASIFT algorithm is quite obvious. Secondly, the feature points matched by SURF algorithm are better than those matched by SIFT algorithm. The number of points also changes significantly. The number of feature points matched of the image processed by homomorphic filtering does not change significantly.

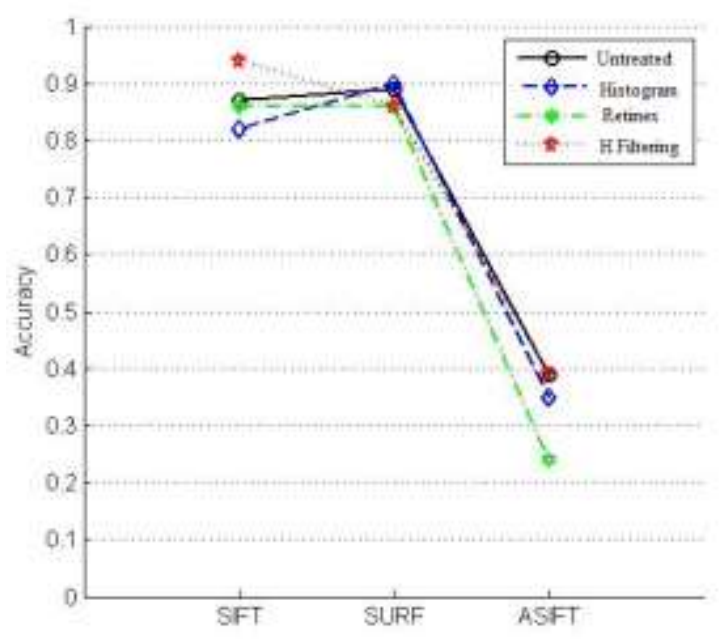

Fig. 5: Matching rate of blurred image by SIFT, SURF and ASIF. 
Fig. 5 shows the matching rate of the feature points of the blurred image. From Fig. 5, it can be concluded that the matching rate of feature points of image by extracted by SURF is higher, which is equalized by histogram. The preprocessing image by homomorphic filtering has higher matching rate of extracting image feature points by SIFT algorithm.

\section{Conclusions}

In this paper, three methods of image enhancement are studied in detail, and three methods of image enhancement are realized through experiments, which improve the number of image feature points matching. The experimental results show that the methods are very effective for image enhancement.

\section{References}

[1] X. Wang, L. Zhang, M. Liu, Y. Li, W.Y. Ma. Arista-image search to annotation on billions of web photos. IEEE Conference Computer Vision and Pattern Recognition, IEEE, 2010, 2987-2994.

[2] L. Cheng, J. Gong, M. Li, Y. Liu. 3D building model reconstruction from multi-view aerial imagery and lidar data. Photogramm Eng Remote Sens. 2011,77 (2):125-139.

[3] W. Freeman, E. Adelson. The design and use of steerable filers. IEEE Transactions Pattern Analysis and Machine Intelligence. 1991, 13 (9): 891-960.

[4] L. Gool, T. Moons, D. Ungureanu. Affine/photometric invariants for planar intensity patterns. Lecture Notes in Computer Science. 1996, 1064: 642-651.

[5] F. Schaffalitzky, A. Zisserman. Multi-view Matching for Unordered Image Sets, or "How Do I Organize My Holiday Snaps?". Lecture Notes in Computer Science. 2002, 2350: 414-431.

[6] S. Belongie, J. Malik, J. Puzicha. Shape matching and object recognition using shape contexts. IEEE Transactions On Pattern Analysis and Machine Intelligence. 2002, 24 (4): 509-522.

[7] D.G. Lowe. Distinctive image features from scale-invariant key points. International Journal of Computer Vision. 2004, 60 (2): 91-110.

[8] H. Bay, T. Tuytelaars, L. Gool. SURF: Speeded Up Robust Features. Computer Vision and Image Understanding. 2008, 110 (3): 346-359.

[9] J. Morel, G. Yu. ASIFT: A new framework for fully affine invariant image comparison. SIAM Journal on Imaging Sciences. 2009, 2: 438-469.

[10] L. Liang, W. He, L. Lei, W. Zhang, H. Wang. Survey on enhancement methods for non-uniform illumination image. Application Research of Computers. 2010, 27 (5):1625-1628. 